\title{
Avaliação de desempenho e capacidade do sistema de saúde espanhol na pandemia de SARS-CoV-2
}

\author{
Evaluation of performance and capacity of the Spanish health system in the SARS-CoV-2 \\ pandemic \\ Evaluación del desempeño y capacidad del sistema de salud español en la pandemia SARS-CoV-2
}

Recebido: 11/01/2021 | Revisado: 13/01/2021 | Aceito: 23/01/2021 | Publicado: 24/01/2021

\author{
Liana de Oliveira Barros \\ ORCID: https://orcid.org/0000-0001-9935-8122 \\ Universidade Estadual do Ceará, Brasil \\ E-mail: lianabarross@gmail.com \\ Francisco Gildemir Ferreira da Silva \\ ORCID: https://orcid.org/0000-0002-5890-3769 \\ Universidade Federal do Ceará, Brasil \\ E-mail:gildemir@gmail.com \\ Bruno de Athayde Prata \\ ORCID: https://orcid.org/0000-0002-3920-089X \\ Universidade Federal do Ceará, Brasil \\ E-mail: baprata@ufc.br
}

\begin{abstract}
Introdução
A Espanha foi um dos países mais afetados pela pandemia de SARS-CoV-2. Embora a saúde pública espanhola seja reconhecida como um dos melhores sistemas de saúde europeus, o pico de casos em um curto período prejudicou o sistema mencionado acima. A avaliação de desempenho possui papel fundamental na gestão dos sistemas de saúde, apresentando indicadores para o apoio à decisão a curto e longo prazo para os planejadores. Metodologia: Este estudo utilizou pesquisa documental do banco de dados públicos do sistema de saúde espanhol e DEA para avaliar a eficiência do atendimento prestado. As etapas para avaliar o impacto do SARS-CoV-2 na eficiência do sistema de saúde são descritas por uma estimativa da eficiência do SARS-CoV-2, seguida por uma comparação das eficiências decorrentes de simulação de cenários factíveis e das reduções de folgas de insumos durante o período demonstrando evento semana a semana utilizando uma linha de base. Resultados: As ações coordenadas para expandir a capacidade do sistema podem ser apresentadas, levando em consideração que insumos críticos podem contrariar o sistema de saúde da Espanha. Identificou-se que o número de enfermeiros foi considerado um insumo crítico. Se esse insumo não tiver tamanho adequado, o sistema de saúde poderá entrar em colapso em curto prazo. Considerações finais: As políticas de expansão no sistema de saúde foram observadas como modelo parcimonioso e com poucas contribuições, uma vez que o sistema de saúde utiliza números relativos de quantidades ideais de dispositivos para profissionais.
\end{abstract}

Palavras-chave: Infecções por Coronavirus; Análise de desempenho; Sistemas de saúde.

\begin{abstract}
Introduction: Spain was one of the countries most affected by the SARS-CoV-2 pandemic. Although Spanish public health is recognized as one of the best European health systems, the peak of cases in a short period has damaged the system mentioned above. Performance evaluation has a fundamental role in the management of health systems, presenting indicators to support short and long-term decision support for planners. Methodology: This study used documentary research from the public database of the Spanish health system and DEA to assess the efficiency of the care provided. The steps to assess the impact of SARS-CoV-2 on the efficiency of the healthcare system are described by an estimate of the efficiency of SARS-CoV-2, followed by a comparison of efficiency simulated feaseble sceneries and reductions in inlet clearances during the period demonstrating an event week by week using a baseline. Results: Coordinated actions to expand the capacity of the system can be presented, taking into account that critical entries may contradict Spain's health system. It was identified that the number of nurses was considered a critical entry. If this entry is not of adequate size, the health care system may collapse in the short term. Final considerations: The expansion policies in the health system were observed as a parsimonious model and with few contributions, since the health system uses relative numbers of ideal amounts of devices for professionals.
\end{abstract}

Keywords: Coronavirus infections; Performance analysis; Health systems.

\section{Resumen}

Introducción: España fue uno de los países más afectados por la pandemia del SARS-CoV-2. Si bien la salud pública española está reconocida como uno de los mejores sistemas sanitarios europeos, el pico de casos en un corto período 
ha dañado el sistema mencionado anteriormente. La evaluación del desempeño tiene un papel fundamental en la gestión de los sistemas de salud, presentando indicadores para apoyar el apoyo a las decisiones a corto y largo plazo de los planificadores. Metodología: Este estudio utilizó una investigación documental de la base de datos pública del sistema de salud español y la DEA para evaluar la eficiencia de la atención brindada. Los pasos para evaluar el impacto del SARS-CoV-2 en la eficiencia del sistema de salud se describen mediante una estimación de la eficiencia del SARS-CoV-2, seguida de una comparación de la eficiencia y las reducciones en los espacios libres de entrada durante el período que demuestra un evento. semana a semana utilizando una línea de base. Resultados: Se pueden presentar acciones coordinadas para ampliar la capacidad del sistema, teniendo en cuenta que las entradas críticas pueden contradecir el sistema sanitario español. Se identificó que el número de enfermeras se consideró un ingreso crítico. Si esta entrada no tiene el tamaño adecuado, el sistema de salud puede colapsar en el corto plazo. Consideraciones finales: Las políticas de expansión en el sistema de salud se observaron como un modelo parsimonioso y con pocos aportes, ya que el sistema de salud utiliza números relativos de cantidades ideales de dispositivos para los profesionales.

Palabras clave: Infecciones por Coronavirus; Análisis de rendimiento; Sistemas de salud.

\section{Introdução}

Após sua descoberta na China em 2019, o SARS-CoV-2 está enfrentando os sistemas de saúde pública de vários países do mundo. A Espanha foi um dos países mais afetados pela pandemia de SARS-CoV-2, apresentando até abril de 2020 um número de 240000 casos com 25.000 mortes, segundo dados oficiais. Embora a saúde pública espanhola seja reconhecida como um dos melhores sistemas de saúde europeus, o pico de casos em um curto período prejudicou o sistema mencionado acima.

Em um período de pandemia, os recursos seriam maiores do que o necessário e a redução da folga implicaria um melhor uso dos recursos. A avaliação de desempenho possui um papel fundamental na gestão dos sistemas de saúde, apresentando indicadores para o apoio à decisão a curto e longo prazo para os planejadores.

Os modelos de Economia da produção têm sido utilizados nas últimas décadas como ferramentas de apoio à decisão para a avaliação de desempenho desses sistemas, levando a benefícios gerenciais expressivos. Neste artigo, abordou-se uma avaliação de desempenho do sistema de saúde espanhol em resposta à pandemia de SARS-CoV-2.

A esse respeito, apresentamos um modelo DEA orientado para a saúde a fim de medir a capacidade e a utilização desta para calcular a necessidade de recursos (médicos, enfermeiros e leitos) na presença de uma pandemia simulando redução de folgas para cada comunidade autônoma, mostrando a volume de recursos que começou a ser mais bem utilizado a partir do aumento da demanda. O pressuposto de adotar o modelo BCC orientado para a produção é a redução da distância dos recursos atuais à fronteira eficiente de Pareto, implicando a redução de excedentes para a determinação de unidades eficientes de tomada de decisão. Além disso, essa abordagem destaca algumas políticas para aumentar a eficiência do sistema.

O restante deste artigo está estruturado da seguinte forma: Primeiramente apresenta-se algumas abordagens relacionadas; Em seguida, descreve-se a metodologia; após, será feita a apresentação de um estudo de caso no sistema de saúde espanhol; e finalmente, a abordagem das principais conclusões, bem como os caminhos de pesquisa para estudos futuros.

A economia da produção é um ramo da economia aplicada na qual uma determinada atividade (produção ou serviço) é analisada considerando o uso de insumos, como trabalho, capital e materiais. As unidades de tomada de decisão (DMUs) são as partes interessadas que participam do processo de decisão do gerenciamento desses recursos. Uma análise da combinação de vários insumos para produzir produtos fornece uma compreensão da eficiência de um determinado processo.

Vários métodos foram usados para medir a produtividade e a eficiência; dentre eles, podemos destacar a Análise Envoltória de Dados - DEA. Em um estudo de 1978, um modelo de programação linear (CCR - Acrônimos de Charnes, Cooper e Rhodes, intercambiável com CRS - retornos constantes de escala) foi introduzido no qual várias unidades de 
produção que executam as mesmas tarefas e apresentam objetivos semelhantes são comparadas em termos de uso de insumos e outputs. (Charnes, 1978). Dessa forma, podemos determinar uma fronteira de eficiência na qual definimos como eficientes as DMUs que operam nessa fronteira; caso contrário, definimos as DMUs como ineficientes. Que o modelo BCC Acrônimos Banker, Charnes e Cooper, intercambiáveis com retornos variáveis de escala do VRS - é o modelo mais frequentemente utilizado em aplicações na área da saúde, com foco especial em hospitais.

O DEA orientado a insumos é usado para determinar quanto de insumos uma empresa poderia contratar se usado com eficiência, a fim de atingir o mesmo nível de produto. Aplicado à medição de capacidade, o DEA orientado a insumos executa o modelo para fatores fixos de produção.

O orientado para o produto é configurado para determinar o produto potencial de uma empresa, considerando seus insumos, se ele operasse eficientemente como empresas ao longo da fronteira de melhores práticas. Uma abordagem orientada para o produto é geralmente mais apropriada para a estimativa da capacidade e utilização da capacidade e, a partir dessa perspectiva, os resultados enfatizam a possibilidade de apoiar uma maior demanda (de casos da doença) por quantidades fixas de recursos (Kohl et al., 2018)

Alguns estudos apresentaram aplicações dos métodos DEA em sistemas de saúde. Podemos destacar as contribuições de no estudo de métodos alternativos para examinar a eficiência hospitalar. Os autores apresentados descrevem como os sistemas de saúde podem melhorar seu desempenho empregando ferramentas DEA (Galterio et al.,2009). Outros apresentaram algumas inovações nos modelos de DEA expressos pela estimativa da eficiência técnica dos sistemas públicos de saúde dos estados brasileiros, utilizando dados demográficos e socioeconômicos não-discricionários. impactos das características na eficiência técnica da oferta de saúde pública (Benegas et al., 2014)

A respeito da eficiência dos hospitais públicos e privados da Andaluzia, Espanha, alguns autores estudaram a eficiência comparativa dos sistemas de saúde de 17 comunidades autônomas e 2 cidades autônomas da Espanha, considerando dados de 2012.10 Um estudo apresentou uma revisão sistemática da literatura sobre a aplicação do DEA em atenção primária à saúde (Herrero et al., 2015)

Em um estudo que utilizou pesquisa documental e DEA para avaliar a eficiência do atendimento prestado pelo SUS, não houve discrepância significativa em relação aos escores de eficiência. O artigo afirma que os recursos físicos disponíveis no SUS foram utilizados de forma satisfatória, contribuindo para a qualidade de vida da população no local pesquisado (Schuster HÁ, 2018)

Outro estudo utilizando a Análise Envoltória de Dados (DEA) para gerar um indicador de eficiência técnica para os cuidados primários, conclui que fatores externos, como analfabetismo, por exemplo, podem influenciar a eficiência da Atenção Primária à Saúde de maneira positiva e negativa, sugerindo que na Atenção Primária à Saúde a gerência deve trabalhar nesses fatores externos para alcançar melhores indicadores de resultados (Dias Cabral et al., 2019).

A abordagem de lanches pode ser usada em estudos de eficiência da capacidade instalada. Por esse período, o lanche refere-se ao tempo extra no contexto organizacional para o desenvolvimento das atividades da instituição, aprendendo e cumprindo os prazos de acordo com.

O Slack pode representar riscos para a organização e pode ser usado para se concentrar em atividades que exigem isso, como inovação. O setor de saúde exige cada vez mais recursos para investimentos em tecnologia e inovação, sendo um dos que mais se beneficiam com a abordagem slack, no entanto, alguns autores consideram uma maneira de desperdiçar recursos que não agregam resultados (Tan et al., 2003; Campos et al.,2016)

Os estudos de eficiência na gestão de recursos financeiros públicos são um dos principais pilares do estado de bemestar social. Um estudo analisou a eficiência dos recursos públicos que os governos regionais da Espanha (Comunidades Autônomas (CA)) investem nos sistemas de saúde (ES) e concluiu que o DEA é um método apropriado para avaliar a 
eficiência dos sistemas de saúde e ajustar a aplicação de fatores econômicos, sociais e políticas organizacionais para melhorar suas eficiências.

A Espanha é um país transcontinental, com a maior parte de seu território localizado no sul da Europa. A Espanha tem aproximadamente 47 milhões de habitantes e uma área de aproximadamente $506.000 \mathrm{~km} 2$. Sua alta densidade populacional associada ao grande número de turistas recebidos todos os anos exige um sistema de saúde robusto para apoiar espanhóis e estrangeiros (Borkan et al., 2010; Cervero et al.2015). Administrativamente, o país está organizado em dezessete comunidades autônomas que possuem um certo grau de autonomia; no entanto, eles estão subordinados a um governo central.

O sistema de saúde pública espanhol tem suas origens no início do século XX. A expansão contínua do sistema para toda a população espanhola foi realizada nas próximas décadas até o final da década de 1980. Na década de 1990, políticas neoliberais foram realizadas na Espanha e o sistema público de saúde reduziu seu alcance. Com a aprovação da Lei 15/1997, novas formas administrativas para centros de saúde foram permitidas e novos hospitais privados foram construídos com recursos públicos.

Atualmente, o sistema de saúde espanhol é composto por instituições públicas (com responsabilidade nacional e centros de saúde apoiados pelas comunidades autônomas) e instituições privadas. Em geral, os seguintes serviços: atendimento primário, atendimento especializado e tratamento de emergência.

Os sistemas de saúde podem melhorar seu desempenho empregando as ferramentas DEA.5 Algumas inovações nos modelos de DEA foram demonstradas em um estudo de 2014, expresso pela estimativa da eficiência técnica dos sistemas públicos de saúde nos estados brasileiros, usando dados demográficos não discricionários e impactos socioeconômicos características da eficiência técnica da prestação de saúde pública (Jacoobs, 2001)

Em 2015, alguns autores avaliaram a eficiência de hospitais públicos e privados na Andaluzia, Espanha. Estudou-se a eficiência comparativa dos sistemas de saúde de 17 comunidades autônomas e 2 cidades autônomas da Espanha, considerando dados de 2012. (Benegas et al., 2014) Foi realizada uma revisão sistemática da literatura em 2020 sobre a aplicação do DEA na atenção primária à saúde.

Por causa do exposto, podemos observar que os estudos que abordam a análise de eficiência dos sistemas de saúde espanhóis são bastante limitados. Além disso, os estudos que abordam a eficiência dos cuidados de saúde em cenários de pandemia também são bastante limitados. Até onde sabemos, não há contribuições relacionadas à análise de eficiência dos sistemas de saúde na pandemia de SARS-CoV-2.

\section{Metodologia}

Apresentamos uma análise prospectiva, na qual hipóteses devem ser assumidas para entender a resposta do sistema de saúde ao comprometimento da capacidade. Seguindo o Kohl et. al (2018) e os aspectos técnicos descritos em Färe, Grosskopf e Lovell (1994) as etapas para avaliar o impacto do COVID na eficiência do sistema de saúde foram delineadas pela estimativa de eficiência ex-ante do COVID, seguida por uma comparação de eficiência do sistema, dada simulação de crescimento da taxa fixa de ocupação por região, e reduções de folga de insumos durante o evento semana a semana usando uma linha de base. As variáveis de insumo foram escolhidas a partir da análise de correlação tradicional usada em modelos DEA. O modelo BCC orientado para a produção foi usado por ser utilizado para mensurar a utilização da capacidade. Desta feita, a proposta metodológica foi dividida nas cinco etapas abaixo:

Primeiro passo: Investigar as variáveis a serem usadas para modelar a eficiência ex ante e as folgas do sistema de saúde em uma situação típica que serve como linha de base. 
Segundo passo: Investigar estimativas ou projeções das taxas de ocupação e hospitalização do evento. Estas estimativas servirão para delimitar cenários de uso do sistema de saúde. Valores comparativos de regiões semelhantes podem ser adotados em indicadores socioeconômicos ou modelos epidêmicos. A estimativa será usada para a etapa três e caberá a um comitê ou grupo avaliar as taxas de projeção mais apropriadas. Note-se que os analistas podem usar duas ou três taxas para uma análise de sensibilidade de cenários otimistas e pessimistas das políticas adotadas para reduzir a propagação da epidemia.

Terceiro passo: Estimar a eficiência do sistema de saúde com cenários simulados de eventos, usando as taxas de ocupação do sistema, conforme prospectado e escolhido no passo dois com as variáveis identificadas no primeiro passo.

Quarta etapa: calcular o índice de ineficiência como inverso do índice de eficiência obtido na etapa três para identificar quando o sistema precisa ser atualizado. O critério de intervenção $a d$ hoc utilizado foi o índice de ineficiência entre 0,5 a 1 como região a ser aprimorada e entre 0,25 a 0,5 como região a ser observada. $\mathrm{O}$ critério poderia ser melhorado se tivéssemos estudado outras intervenções em lugares diferentes ao redor da palavra, como não tínhamos, então realizamos um propósito ad hoc.

Quinta etapa: Usar as folgas dos cenários simulados para compreender os insumos que são críticos no sistema conforme a taxa de ocupação muda e que exigirão ampliação ou realocação. De acordo com o modelo DEA usado e sua orientação, as folgas indicam os insumos que devem ser melhorados (Ver Beck \& Beuren, 2015; Mallidou, et. Al., 2011).

A escolha da metodologia foi baseada em Färe, Grosskopf e Lovell (1994), considerando também as armadilhas destacadas por Kohl et. al (2018), como: Seleção de dados é o primeiro passo no processo de modelagem, o analista deve procurar fatores que caracterizem o processo analisado da melhor forma possível. maneira de incorporar insumos e produtos que descrevem os objetivos gerenciais da DMU; A especificação do modelo é parte importante do processo, mas mesmo com uma diversificação permanente dos modelos DEA, os modelos básicos da DEA são os modelos mais frequentemente utilizados em hospitais e serviços de saúde e em um novo procedimento metodológico, conforme fornecido neste documento, o BCC ou Modelo de CCR são adequados; Tentou-se incorporar o efeito de congestionamento na análise da DEA, mas os debates acadêmicos não terminaram, então não o realizamos; Técnicas subseqüentes podem ser exploradas, mas o ganho de precisão não se justifica até que pesquisas teóricas e empíricas adicionais produzam resultados mais robustos.

No estudo de caso, as etapas para avaliar o impacto do SARS-CoV-2 na eficiência do sistema de saúde são descritas por uma estimativa da eficiência do SARS-CoV-2, seguida por uma comparação da eficiência e das reduções de folgas de insumos durante o período. Evento semana a semana usando uma linha de base.

Assumimos uma taxa fixa de ocupação do sistema de saúde por região devido à falta de informações precisas. Além disso, adotamos as variáveis de insumo da análise de correlação tradicional amplamente usada nos modelos DEA. Os resultados são o número de atendimentos de saúde e o período base é o disponível na operação antes da pandemia. Como nossos interesses são capacidade e utilização de capacidade, aplicamos o conhecido modelo BCC orientado para a produto (Campos-Lucena et al.,2018)

Essa metodologia visa entender o colapso da capacidade do sistema de saúde espanhol e se uma análise de cenário pode ser usada para orientar o aumento do insumo. O resultado das estimativas de liberação e eficiência do sistema servirá como diretrizes para alterações de insumo em resposta ao colapso previsto.

\section{Resultados e Discussão}

O estudo de caso estudado considerou as seguintes DMUs: Andaluzia, Aragão, Astúrias, Principado, Baleares, Ilhas Canárias, Cantábria, Castela e Leão, Castela-La Mancha, Catalunha, Comunidade Valenciana, Extremadura, Galiza, Madri, 
Comunidade de Múrcia, Região de Navarra, C Foral de País Vasco-Euskadi, Rioja, Ceuta e Melilla. Agregamos Ceuta e Melilla como uma única DMU aos dados disponíveis. Todos os dados foram coletados no site do Ministro da Saúde da Espanha.

Para a avaliação de desempenho do sistema de saúde espanhol durante a crise SARS-CoV-2, avaliamos as seguintes variáveis de insumo em potencial: total de profissionais (IM1), equipamento de atenção primária (IM2), número de médicos por hospital (IM3), número de médicos em serviços de emergência e emergência (IM4), número de profissionais médicos especializados (IM5), total de profissionais médicos por 1.000 habitantes (IM6), total de profissionais de enfermagem (IE1), equipamentos de atenção primária (IE2), número de enfermeiros por hospital ( IE3), número de enfermeiros em serviços de emergência e emergência (IE4), número de profissionais de saúde em treinamento especializado (IE5), total de profissionais de enfermagem por 1.000 habitantes (IE6), número de hospitais (IC1), número de leitos hospitalares (IC2) Postos de Hospitais - PHD (IC3), leitos por 1.000 habitantes (IC4), PHD por 100.000 habitantes (IC5). Como produto, consideramos o número de casos de SARS-CoV-2 (P1). São resumidas as estatísticas na Tabela 1.

Tabela 1. Variáveis estatísticas.

\begin{tabular}{|c|c|c|c|c|}
\hline Variável & Média & Desvio Padrão & Min & Max \\
\hline IM1 & 8149,00 & 7462,00 & 391,00 & 23947,00 \\
\hline IM2 & 1974,00 & 1790,00 & 94,00 & 6144,00 \\
\hline IM3 & 4573,00 & 4148,00 & 252,00 & 13909,00 \\
\hline IM4 & 180,40 & 170,30 & 20,00 & 720,00 \\
\hline IM5 & 1421,00 & 1450,00 & 25,00 & 5134,00 \\
\hline IM6 & 3,19 & 0,39 & 2,30 & 3,70 \\
\hline IE1 & 10099,00 & 8715,00 & 592,00 & 29431,00 \\
\hline IE2 & 1694,00 & 1478,00 & 82,00 & 5398,00 \\
\hline IE3 & 8124,00 & 7043,00 & 482,00 & 23300,00 \\
\hline IE4 & 170,10 & 158,40 & 10,00 & 632,00 \\
\hline IE5 & 111,40 & 99,87 & 4,00 & 332,00 \\
\hline IE6 & 4,12 & 0,54 & 3,30 & 5,30 \\
\hline IC1 & 25,89 & 33,93 & 2,00 & 156,00 \\
\hline IC2 & 6200,00 & 6018,00 & 340,00 & 25266,00 \\
\hline IC3 & 1014,00 & 1250,00 & 53,00 & 5216,00 \\
\hline IC4 & 2,49 & 0,49 & 1,70 & 3,40 \\
\hline IC5 & 36,63 & 11,58 & 23,50 & 70,00 \\
\hline P1 & 5852,00 & 10869,00 & 0,00 & 60487,00 \\
\hline
\end{tabular}

Fonte: Elaborada pelos autores. 
Consideramos os valores das variáveis de insumo no sistema de saúde espanhol antes da crise da SARS-CoV-2. Além disso, não consideramos hospitais de campo em nossa análise, pois essas informações não estavam disponíveis para os pesquisadores. A análise foi realizada com o índice de contaminação da SARS-CoV-2 no seguinte período de oito semanas: 19/3/2020, 26/3/2020, 2/2/2020, 04/09/2020, 16/4 / 2020 e 23/04/2020.

Montamos casos de SARS-CoV-2 semanas a semana como hospitalização e até 2018 (pontos base) considerando o número de casos de SARS-CoV-2 na semana 23/04/2020. As internações foram assumidas homogêneas para todas as DMUs, no entanto, é possível simular diferenciações por unidades autônomas, não sendo realizada pela falta de informações sobre aspectos regionais e por não termos modelos epidemiológicos para encaminhamento de casos.

Cabe ressaltar que os autores apontam que os aspectos regionais são importantes para uma melhor mensuração das eficiências (Benegas et al., 2014) As premissas implícitas foram a ocorrência do SARS-CoV-2 da mesma maneira em todas as regiões autônomas e que todo o sistema foi utilizado para combater a pandemia.

Se não tivéssemos relatado casos de SARS-CoV-2, poderíamos realizá-lo com simulações do crescimento da hospitalização e adicioná-lo a um período base. A análise de correlação, apresentada na Tabela 2 pelo mapa de calor, indica que o total de profissionais médicos por 1.000 habitantes e o total de profissionais enfermeiros por 1.000 habitantes (IM6 e IE6), bem como o número de leitos hospitalares (IC5), podem ser utilizados como variáveis de insumo, pois eles apresentam forte relação entre eles em comparação com outras variáveis (Banker et al.,1984). 
Tabela 2. Mapa de calor das variáveis.

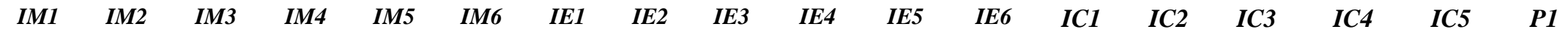

\begin{tabular}{|c|c|c|c|c|c|c|c|c|c|c|c|c|c|c|c|c|c|c|}
\hline IM1 & 1 & 1 & 1 & 0,8 & 1 & 0,0 & 1 & 1 & 1 & 0,8 & 1 & $-0,4$ & 0,8 & 0,9 & 0,9 & $-0,1$ & 0,3 & 0,2 \\
\hline IM2 & 1 & 1 & 1 & 0,8 & 0,94 & $-0,1$ & 1 & 1 & 1 & 0,9 & 1 & $-0,4$ & 0,8 & 0,9 & 0,9 & $-0,1$ & 0,3 & 0,2 \\
\hline IM3 & 1 & 1 & 1 & 0,8 & 1 & 0,0 & 1 & 1 & 1 & 0,8 & 1 & $-0,4$ & 0,8 & 1 & 0,92 & $-0,1$ & 0,3 & 0,2 \\
\hline IM4 & 0,8 & 0,8 & 0,8 & 1 & 0,7 & $-0,2$ & 0,8 & 0,8 & 0,8 & 1 & 0,8 & $-0,5$ & 0,5 & 0,7 & 0,6 & $-0,3$ & 0,0 & 0,3 \\
\hline IM5 & 1 & 0,94 & 1 & 0,7 & 1 & 0,0 & 1,0 & 0,9 & 1,0 & 0,8 & 1,0 & $-0,4$ & 0,7 & 0,9 & 0,9 & $-0,2$ & 0,3 & 0,2 \\
\hline IM6 & 0,0 & $-0,1$ & 0,0 & $-0,2$ & 0,0 & 1 & $-0,1$ & $-0,1$ & 0,0 & $-0,2$ & 0,0 & 0,7 & 0,0 & 0,0 & 0,0 & 0,5 & 0,3 & 0,1 \\
\hline IE1 & 1 & 1 & 1 & 0,8 & 1 & $-0,1$ & 1 & 1 & 1 & 0,8 & 1 & $-0,4$ & 0,8 & 1 & 0,9 & $-0,1$ & 0,3 & 0,2 \\
\hline IE2 & 1 & 1 & 1 & 0,8 & 0,9 & $-0,1$ & 1 & 1 & 1 & 0,9 & 0,9 & $-0,4$ & 0,8 & 1 & 0,9 & $-0,1$ & 0,3 & 0,2 \\
\hline IE3 & 1 & 1 & 1 & 0,8 & 1 & 0,0 & 1 & 1 & 1 & 0,8 & 1 & $-0,4$ & 0,8 & 0,9 & 0,9 & $-0,1$ & 0,3 & 0,2 \\
\hline IE4 & 0,8 & 0,9 & 0,8 & 1 & 0,8 & $-0,2$ & 0,8 & 0,9 & 0,8 & 1 & 0,83 & $-0,5$ & 0,6 & 0,8 & 0,7 & $-0,3$ & 0,1 & 0,4 \\
\hline IE5 & 1 & 1 & 1 & 0,8 & 1,0 & 0,0 & 1 & 0,94 & 1 & 0,8 & 1 & $-0,4$ & 0,7 & 0,9 & 0,9 & $-0,2$ & 0,2 & 0,2 \\
\hline IE6 & $-0,4$ & $-0,4$ & $-0,4$ & $-0,5$ & $-0,4$ & 0,7 & $-0,4$ & $-0,4$ & $-0,4$ & $-0,5$ & $-0,4$ & 1 & $-0,2$ & $-0,3$ & $-0,3$ & 0,6 & 0,3 & $-0,2$ \\
\hline IC1 & 0,8 & 0,8 & 0,8 & 0,5 & 0,7 & 0,0 & 0,8 & 0,8 & 0,8 & 0,6 & 0,7 & $-0,2$ & 1 & 0,9 & 1,0 & 0,3 & 0,6 & 0,3 \\
\hline IC2 & 0,9 & 0,9 & 1 & 0,7 & 0,9 & 0,0 & 1 & 1,0 & 0,9 & 0,8 & 0,9 & $-0,3$ & 0,94 & 1 & 1 & 0,1 & 0,5 & 0,3 \\
\hline IC3 & 0,9 & 0,9 & 0,92 & 0,6 & 0,9 & 0,0 & 0,9 & 0,9 & 0,9 & 0,7 & 0,9 & $-0,3$ & 1 & 1 & 1 & 0,1 & 0,6 & 0,3 \\
\hline IC4 & $-0,1$ & $-0,1$ & $-0,1$ & $-0,3$ & $-0,2$ & 0,5 & $-0,1$ & $-0,1$ & $-0,1$ & $-0,3$ & $-0,2$ & 0,6 & 0,3 & 0,1 & 0,1 & 1 & 0,4 & 0,2 \\
\hline IC5 & 0,3 & 0,3 & 0,3 & 0,0 & 0,3 & 0,3 & 0,3 & 0,3 & 0,3 & 0,1 & 0,2 & 0,3 & 0,6 & 0,5 & 0,6 & 0,4 & 1 & 0,1 \\
\hline P1 & 0,2 & 0,2 & 0,2 & 0,3 & 0,2 & 0,1 & 0,2 & 0,2 & 0,2 & 0,4 & 0,2 & $-0,2$ & 0,3 & 0,3 & 0,3 & 0,2 & 0,1 & 1 \\
\hline
\end{tabular}

Fonte: Elaborada pelos autores. 
Estimamos a eficiência e calculamos o índice ineficiente conforme indicado do primeiro ao quarto passo descrito anteriormente. Para testar a robustez dos resultados, realizamos um cenário comparativo usando o mesmo modelo e orientação da DEA para o caso real do índice de hospitalização no episódio pandêmico e a média de todos os casos reais do índice de hospitalização por região foi usada como linha de base em 2018.

Na Tabela 3, comparando os resultados do índice ineficiente do cenário real para o cenário de simulação, o caso real apresenta mais uma região autônoma como ineficiente. A partir da linha de base no caso de simulação, a terceira semana (2/4/2020) foi crítica (índice ineficiente de 0,5 a 1) para Illes Canarias, Communidad de Murcia e Ceuta e Melilla. O caso real é igual simulado adicionado à Catalunha.

Tabela 3. Índice ineficiente. REGIONS SIMULATED INEFFICIENCIES REAL INEFFICIENCIES

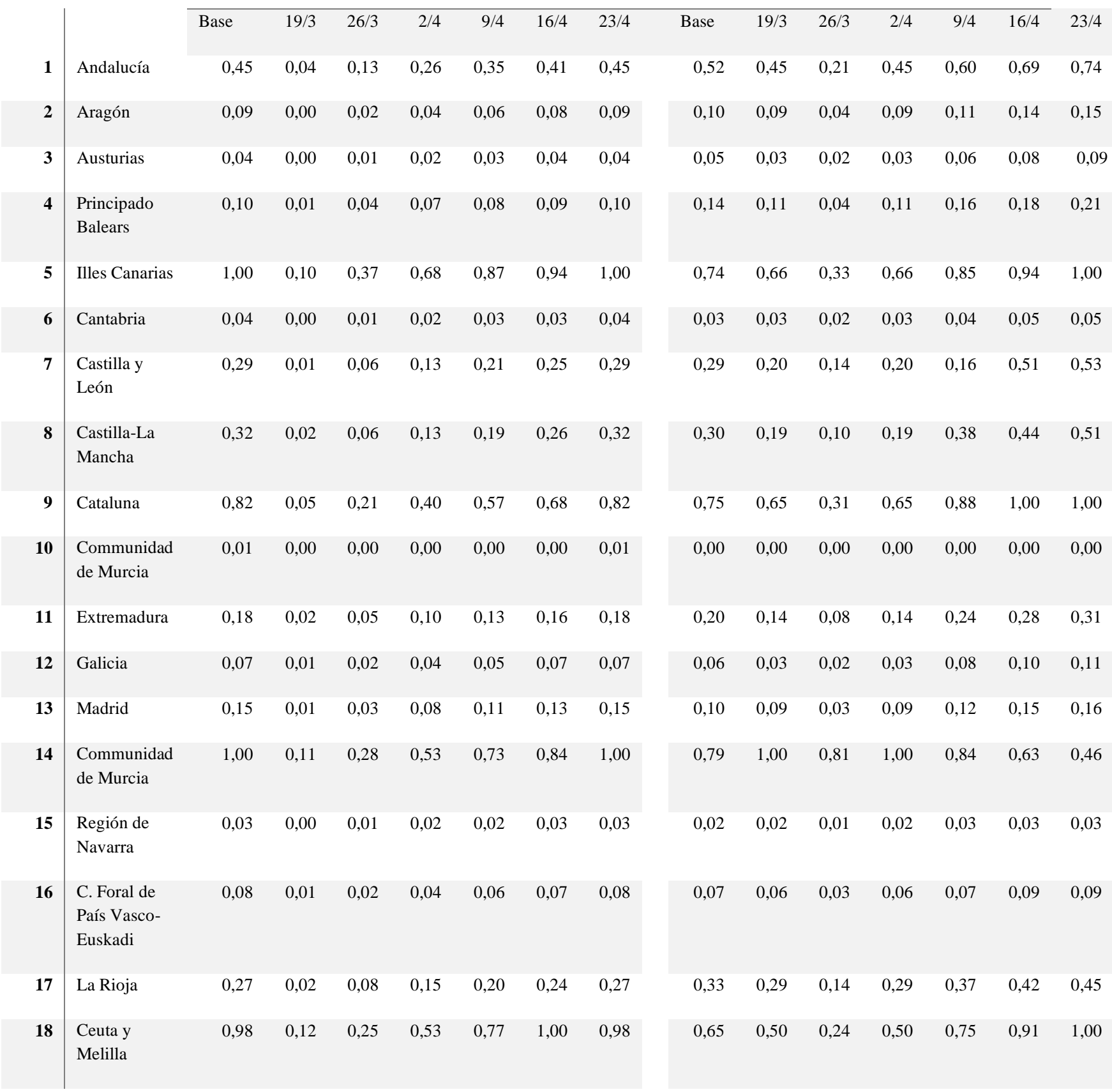




\section{INEFICIÊNCIA ENTRE 0,5 E 1}

\section{INEFICIÊNCIA ENTRE 0,25 E 0,49}

Fonte: Elaborada pelos autores.

Andaluzia, Aragão, Astúrias, Principado dos Baleares, Cantábria, Comunidade de Múrcia, Extremadura, Galiza, Madri, Região de Navarra e C. Foral do País Vasco-Euskadi são DMUs não foram ineficientes durante o caso de simulação. A Catalunha apresenta desempenho ineficiente após a quarta semana, que não era o caso de Madri, mesmo com as mesmas condições de insumos. Supomos que esse resultado tenha ocorrido em simulação pela diferença no número de casos de hospitalizações da Catalunha quase cinco vezes maior que Madri.

Usando o quinto passo, a intervenção pode ocorrer na terceira semana em Illes Canarias, Comunidade de Múrcia e Ceuta e Melilla, porque não foi suficiente pela demanda aumentando em torno da terceira semana, como indica o índice ineficiente. Todos os insumos precisam ser atualizados. Procurando por colegas, descobrimos que Madri é a referência. Usando o par (também em Madri), indicamos o quanto os insumos podem ser melhorados na Tabela 4.

A proporção de melhoria foi calculada multiplicando-se a contribuição do par pelo número de internações na região ineficiente e dividindo o resultado pelo número de hospitalizações no par. Para melhorar os insumos na região ineficiente, usamos a média da proporção calculada mais os insumos na região antes do episódio de pandemia.

Como nossos resultados, exceto Catalunã (Campos et al. 2016), indicam que As Illes Canarias são ineficientes e devem melhorar o uso de insumos analisados pelo mesmo autor: Balears, Castela e Leão, Castela e Mancha, Catalunha e Madri. eficiente.

Quadro 1. Insumos aumentados antes e durante o SARS-COVID-2.

\begin{tabular}{|c|c|c|c|c|c|c|}
\hline & \multicolumn{2}{|c|}{ Ilhas Canarias } & \multicolumn{2}{|c|}{$\begin{array}{l}\text { Comunidade de } \\
\text { Murcia }\end{array}$} & \multicolumn{2}{|c|}{ Ceuta y Melilla } \\
\hline & Antes & Durante & Antes & Durante & Antes & Durante \\
\hline $\begin{array}{l}\text { Total médicos por } 1.000 \text { habitantes } \\
\text { (IM6) }\end{array}$ & 2,7 & 3,28 & 3 & 3,05 & 2,3 & 3,22 \\
\hline $\begin{array}{l}\text { Total enfermeiros por } 1.000 \\
\text { habitantes (IE6) }\end{array}$ & 3,9 & 4,55 & 3,4 & 3,46 & 3,5 & 4,53 \\
\hline Número de leitos de hospitais & 23,5 & 29,66 & 28,2 & 28,8 & 31,2 & 41,02 \\
\hline
\end{tabular}

Fonte: Elaborada pelos autores.

Essa metodologia pode apoiar o desenvolvimento de políticas para expandir os sistemas de saúde em congestionamento. Em outros casos, nos quais a sobrecarga é prevista, pode-se empregar o uso da técnica DEA associada aos cenários de crescimento da demanda.

A proposta é flexível, pois diferentes choques podem ser dados expandindo-se em períodos curtos ou aumentando a intensidade de uso do sistema por meio do percentual de hospitalizações. No estudo de caso, podemos observar que o número de enfermeiros, médicos e leitos hospitalares são gargalos do sistema. No entanto, o número de respiradores ou dispositivos de ventilação seria relevante para indicar como a expansão poderia ser processada. 
Infelizmente, esta informação não está disponível para pesquisadores. Podemos observar que, como o sistema de saúde utiliza um número relativo de quantidades ideais do dispositivo para cada médico e enfermeiro, o número ideal de dispositivos pode ser determinado, pois sabemos o número necessário de profissionais.

\section{Considerações Finais}

Neste estudo, foi apresentada uma metodologia para avaliar quais insumos e quando expandir os insumos de produção dos sistemas de saúde em uma situação de expansão da demanda e congestionamento. A metodologia utiliza técnicas já exploradas na literatura em saúde, com a inovação da aplicação de cenários para o uso da técnica DEA.

O estudo de caso foi desenvolvido para a Espanha, observando a pressão no sistema de saúde deste país e considerando uma taxa estimada de hospitalização. Os resultados indicam que ações coordenadas para expandir a capacidade do sistema podem ser apresentadas, levando em consideração que insumos críticos podem contrariar o sistema.

Na Espanha, identificou-se o número de enfermeiros como um insumo crítico. Se este insumo não tiver tamanho adequado, o sistema de saúde poderá entrar em colapso no curto prazo. A metodologia é flexível e pode ser utilizada sob a perspectiva de choques exógenos no sistema de saúde.

As políticas de expansão no sistema de saúde foram observadas como modelo parcimonioso e com poucas contribuições, uma vez que o sistema de saúde utiliza números relativos de quantidades ideais de dispositivos para profissionais. O uso da técnica deve estar alinhado com as previsões epidemiológicas do crescimento de casos a serem utilizados como previsões da demanda do sistema.

Recomenda-se expandir os recursos para a aquisição de insumos e otimizá-los para enfrentar a pandemia. O trabalho de Carrasco, G. (2020) pode ser um ponto de partida, considerando os aspectos de qualidade apontados e Zakowska, e GodyckiCwirko (2020) indica modelos DEA mais adequados para modelar o congestionamento do sistema de saúde.

\section{Agradecimentos}

Os autores agradecem ao Conselho Nacional de Desenvolvimento Científico e Tecnológico (CNPq) por financiar parcialmente o desenvolvimento deste trabalho com as bolsas 404232 / 2016-7, 303594 / 2018-7 e 422898 / 2018-0.

\section{Referências}

Banker, R. D., Charnes, A., \& Cooper, W. W. (1984). Some models for estimating technical and scale inefficiencies in data envelopment analysis. Management science, 30(9), 1078-1092.

Beck, F., \& Beuren, I. M. (2015). Folga organizacional: Análise em uma perspectiva comportamental no campo empírico. Revista Universo Contábil, 11(4), 626.10.4270/RUC.2015429. htts:// www.redalyc.org/articulo.oa?id=117043928002

Benegas, M., \& Silva, F. G. F. D. (2014). Estimação da eficiência técnica do SUS nos estados brasileiros na presença de insumos não-discricionários. Revista Brasileira de Economia, 68(2), 171-196.

Borkan, J., Eaton, C. B., Novillo-Ortiz, D., Rivero Corte, P., \& Jadad, A. R. (2010). Renewing primary care: lessons learned from the Spanish health care system. Health Affairs, 29(8), 1432-1441.

Campos, M. S., Fernández-Montes, A., Gavilan, J. M., \& Velasco, F. Public resource usage in health systems: a data envelopment analysis of the efficiency of health systems of autonomous communities in Spain. Public Health. 2016; 138, 33-40. 10.1016/j.puhe.2016.03.003

Campos-Lucena, M. S., Sanz-Díaz, M. T., \& Morente, F. V. (2018). The efficiency of health services in Spain based on data envelopment analysis (DEA). The singularity of health efficiency. International Journal of Collaborative Research on Internal Medicine \& Public Health, 10(1).

Carrasco, G. (2020). Reflections on the quality of health care after the SARS-CoV-2 pandemic. Journal of Healthcare Quality Research, 35 (2), 61.

Cervero-Liceras, F., McKee, M., \& Legido-Quigley, H. (2015). The effects of the financial crisis and austerity measures on the Spanish health care system: a qualitative analysis of health professionals' perceptions in the region of Valencia. Health Policy, 119(1), 100-106.

Charnes, A. (1978). Measuring efficiency of decision-making units. European Journal of Operational Research, 2(6), $429-444$. 
Research, Society and Development, v. 10, n. 1, e53410111886, 2021

(CC BY 4.0) | ISSN 2525-3409 | DOI: http://dx.doi.org/10.33448/rsd-v10i1.11886

Dias Cabral, K. F., Ferreira, M. A. M., Batista, R. S., Cerqueira, F. R. Alternate title: Primary health care: an analysis of the technical efficiency of resources in the state of Minas Gerais. Revista de Gestão em Sistemas de Saúde. 8 (2), 137-150.

Färe, R. S. Grosskopf and CAK Lovell (1994). Production Frontiers. Cambridge, UK, Cambridge.

Galterio, L., Helton, J., Langabeer 2nd, J., \& DelliFraine, J. (2009). Data envelopment analysis: performance normalization and benchmarking in healthcare. Journal of healthcare information management: JHIM, 23(3), 38-43.

Herrero, L. T., Martín, J. M., \& López, P. D. A. G. M. (2015). Technical efficiency of traditional hospitals and public enterprises in Andalusia (Spain). Gaceta sanitaria, 29(4), 274-281.

Jacobs, R. (2001). Alternative methods to examine hospital efficiency: data envelopment analysis and stochastic frontier analysis. Health care management science, $4(103-115$.

Kohl, S., \& Schoenfelder, J., \& Fügener, A., \& Brunner, J. (2018). The use of Data Envelopment Analysis (DEA) in healthcare with a focus on hospitals. Health Care Management Science. 22. 10.1007/s10729-018-9436-8.

Mallidou, A. A., Cummings, G. G., Ginsburg, L. R., Chuang, Y. T., Kang, S., Norton, P. G., \& Estabrooks, C. A. (2011). Staff, space, and time as dimensions of organizational slack: A psychometric assessment. Health Care Management Review, 36(3), 252-264. 10.1097/HMR.0b013e318208ccf8. https://pubmed.ncbi.nlm.nih.gov/21646884/

Schuster, H. Á., Muller, S. H., Rodrigues Junior, M. M. Avaliação da Eficiência do Atendimento no Sus por meio da Análise Envoltória de Dados: um Estudo nas Microrregiões do Estado do Paraná. Administração Pública e Gestão Social. 10(3),179-187. 9p.

Tan, J., \& Peng, M. W. (2003). Organizational slack and firm performance during economic transitions: Two studies from an emerging economy. Strategic Management Journal, 24(13), 1249-1263. doi:10.1002/smj.351. Links: https://doi.org/10.1016/j.jbusres.2020.08.038

Zakowska, I., \& Godycki-Cwirko, M. (2020). Data envelopment analysis applications in primary health care: a systematic review. Family Practice, 37(2), 147-153. 